\title{
An inoculum-dependent culturing strategy (IDC) for the cultivation of environmental microbiomes and the isolation of novel endophytic Actinobacteria
}

\author{
Mohamed S. Sarhan $\mathbb{D}^{1,2} \cdot$ Elhussein F. Mourad $\mathbb{D}^{1} \cdot$ Rahma A. Nemr $^{1} \cdot$ Mohamed R. Abdelfadeel $^{1} \cdot$ \\ Hassan-Sibroe A. Daanaa ${ }^{3} \cdot$ Hanan H. Youssef ${ }^{1} \cdot$ Hanan A. Goda $^{1} \cdot$ Mervat A. Hamza ${ }^{1} \cdot$ Mohamed Fayez $^{1}$. \\ Bettina Eichler-Löbermann ${ }^{2} \cdot$ Silke Ruppel $^{4} \cdot$ Nabil A. Hegazi $^{1}$
}

Received: 5 March 2019 / Revised: 25 July 2019 / Accepted: 4 August 2019 / Published online: 29 August 2019

(c) The Author(s) 2019. This article is published with open access

\begin{abstract}
The recent introduction of plant-only-based culture media enabled cultivation of not-yet-cultured bacteria that exceed $90 \%$ of the plant microbiota communities. Here, we further prove the competence and challenge of such culture media, and further introduce "the inoculum-dependent culturing strategy, IDC". The strategy depends on direct inoculating plant serial dilutions onto plain water agar plates, allowing bacteria to grow only on the expense of natural nutrients contained in the administered inoculum. Developed colonies are successively transferred/subcultured onto plant-only-based culture media, which contains natural nutrients very much alike to those found in the prepared plant inocula. Because of its simplicity, the method is recommended as a powerful tool in screening programs that require microbial isolation from a large number of diverse plants. Here, the method comfortably and successfully recovered several isolates of endophytic Actinobacteria represented by the six genera of Curtobacterium spp., Plantibacter spp., Agreia spp., Herbiconiux spp., Rhodococcus spp., and Nocardioides spp. Furthermore, two of the isolates are most likely novel species belonging to Agreia spp. and Herbiconiux spp.
\end{abstract}

Not-yet-cultured populations represent diverse groups of microbes that account for more than $90 \%$ of a given ecosystem that are still under shadow [1]. To gain insights into their unknown functions and exploit their potentials, different approaches were recently introduced to culture such

Supplementary information The online version of this article (https:// doi.org/10.1038/s41429-019-0226-4) contains supplementary material, which is available to authorized users.

$\triangle$ Nabil A. Hegazi

hegazinabil8@gmail.com

1 Department of Microbiology, Faculty of Agriculture, Cairo University, Giza, Egypt

2 Faculty of Agricultural and Environmental Sciences, Rostock University, Rostock, Germany

3 Department of Genetics, School of Life Science, the Graduate University for Advanced Studies (SOKENDAI), 1111 Yata, Mishima, Shizuoka 411-8540, Japan

4 Leibniz Institute of Vegetable and Ornamental Crops (IGZ), Großbeeren, Germany not-yet-cultured bacteria $[2,3]$. In this respect, the plantonly-based culture media were presented as natural culture media to replace myriad formulas of synthetic culture media, and strongly recommended to increase the cultivability of the plant microbiota [4-10]. To alleviate the stress of disproportionate nutrients, present in common culture media, we aimed at culturing maize and sunflower microbiota on the natural nutrients present in the plant inoculum itself, compared to highly diluted plant-only-based culture media and standard R2A. The rationale of the study is to allow the existing plant microbiota to grow on nutrientdeficient water agar (WA) depending on the proportionate nutrients present in the administered inoculum itself. Expressly, the plant inoculum is used as a dual source of enclosed microbes and inherent nutrients.

To test this hypothesis, appropriate serial dilutions of surface-sterilized roots (rhizosphere) and shoots (phyllosphere) of maize plants (Zea mays L.) were surfaceinoculated on plain WA, principally used to examine the growth of colony-forming units (CFUs) of plant endophytes depending on the nutrients contained in the inoculum itself. For comparisons, i.e., bench marking, other sets of agar 
a

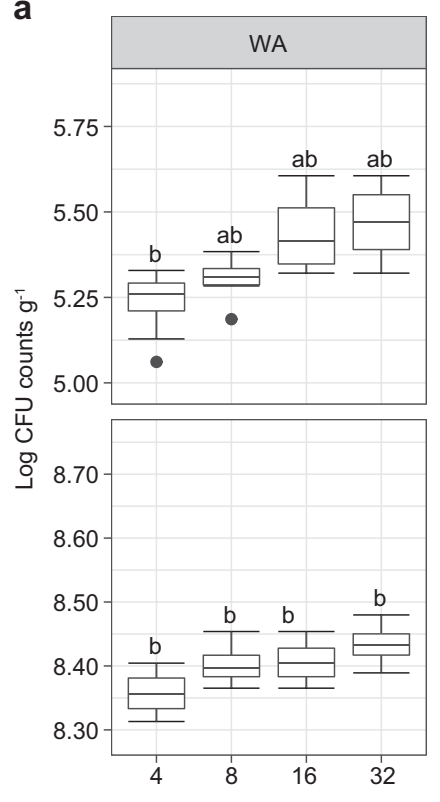

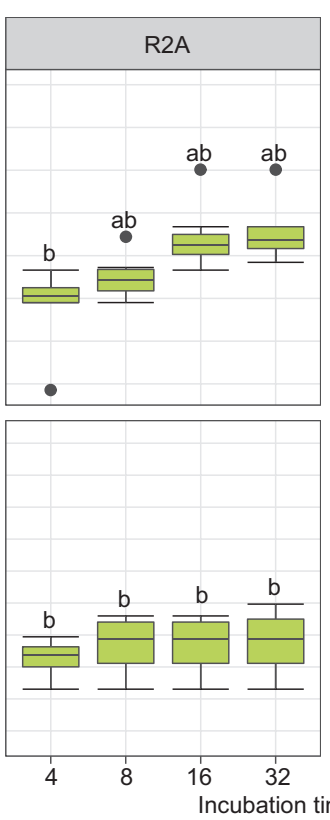

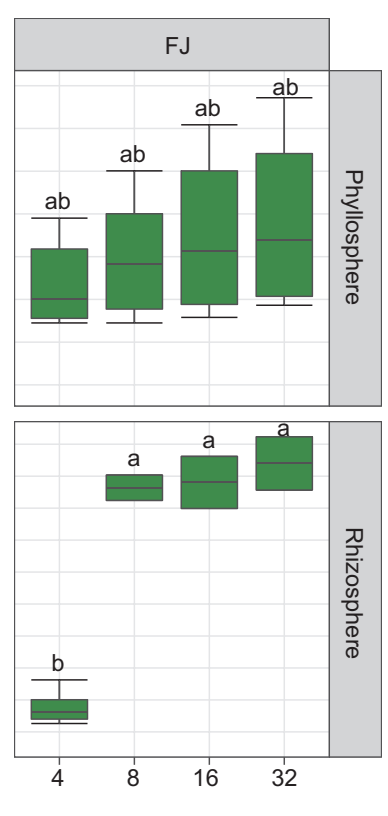

b

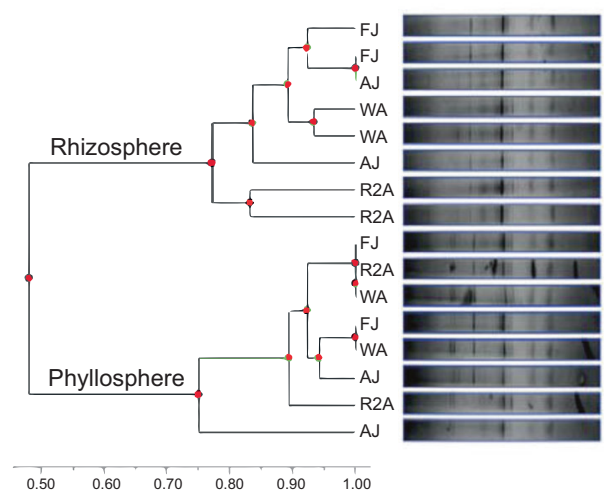

Fig. 1 Culture-dependent bacterial community analysis of maize rhizosphere and phyllosphere. a Log CFU counts developed on the tested culture media over time. Statistically significant differences are designated by different letters based on Tukey's Honestly Significant Differences (HSD, $P \leq 0.05, n=4)$ ). b DGGE analysis of $16 \mathrm{~S}$ rRNA gene profiling of culturable bacterial community recovered on the tested culture media after 32 days of incubation, clustered with
C

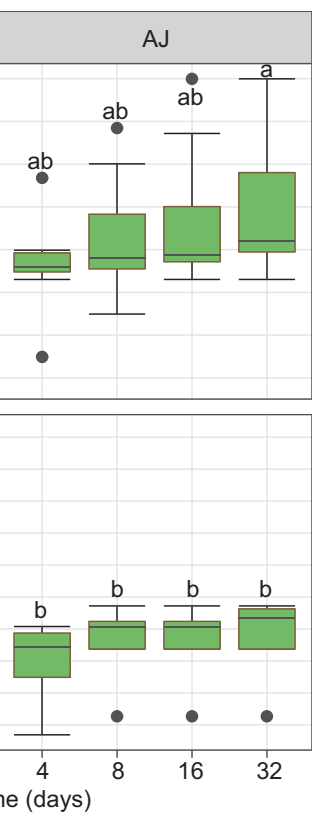

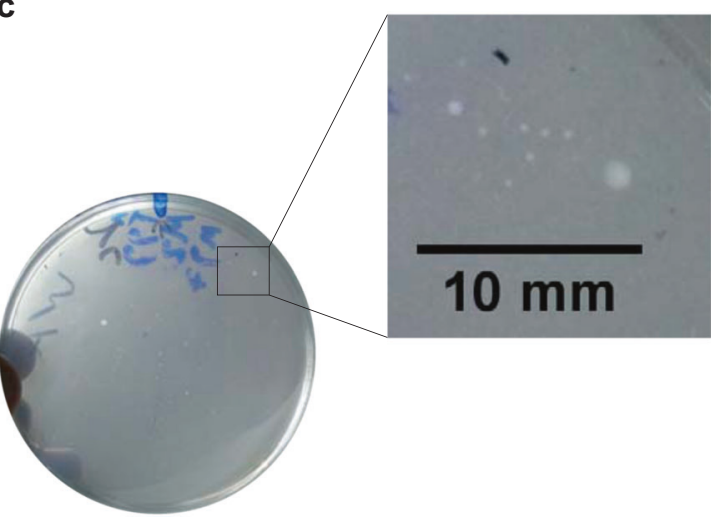

UPGMA method based on Euclidean distances (totallab.com/home/ cliqs). c Morphologies of colonies grown on WA together with zoom in section of macro- and micro-colonies. Scaling was carried out using ImageJ software (imagej.nih.gov/ij). WA water agar, R2A 1/100 diluted (v/v) R2A, AJ autoclaved 1/200 diluted (v/v) maize juice agar, and $\mathrm{FJ}$ filtered $1 / 200$ diluted $(\mathrm{v} / \mathrm{v})$ maize juice agar culture media

counts for either spheres. The extension of incubation time significantly stimulated the development of microcolonies (i.e., $\mu \mathrm{CFU},<1 \mathrm{~mm}$ dia.). Notably, the development of such microcolonies was very much reported for the rhizosphere compartment by the end of the incubation period (32 days), representing $>20 \%$ out of total CFU counts (Fig. S1). Such phenomenon of microcolonies development was brought into focus while introducing novel approaches for increasing culturability of environmental microbiomes [2, 11, 12]. Indeed, the development of microcolonies was significantly enhanced by new methodologies for culturing the uncultured bacterial populations, e.g., the use of overlay agar 
technique for plating [13], the diffusion-chamber-based techniques, encapsulation of cells in gel microdroplets under low nutrients flux conditions, and soil slurry membrane system that combines a polycarbonate membrane as a growth support and soil extract as substrate [14]. Majority of microcolonies developed by such techniques were isolated in pure cultures and identified as not-yet-cultured/ novel species bacteria [14]. The recurrent development of such microcolonies was experienced in the present study, depending on the combined effect of prolonged incubation time and the proportionate nutrient concentrations present either in WA, receiving plant nutrients contained in the plant inoculum itself, or the extensively diluted plant juice and R2A agar. In other words, the limited nutrients, present in such extensively diluted culture media, do not satisfy the growth of fast-growing bacteria, but allow the recovery of fastidious ones developed in form of microcolonies.

Although the limitations of PCR-DGGE of the $16 \mathrm{~S}$ rRNA gene, the method is nonetheless in practice for studying microbial diversity [15]. Consequently, we used culture-dependent PCR-DGGE of the $16 \mathrm{~S}$ rRNA gene to analyze the community compositions of the culturable endophytes of maize rhizosphere and phyllosphere [7, 16]. PCR-DGGE analysis of CFUs harvest clearly differentiated the rhizosphere populations from those inhabiting the phyllosphere at similarity level of $48 \%$ (Fig. 1b). This might be attributed to the differential composition of the nutrient pools of either plant compartments. No explicit separations were reported as due to the culture media effect. Hence, it is suggested that by using highly diluted culture media, the inoculum has stronger impact in shaping culturable community composition than the culture medium by itself. This is in consistency with the findings of Hegazi et al. [6]. They reported gradual separations of DGGE profiles of culturable population of Lucerne rhizobacteria according to nutrient concentrations and origin; at the very low concentrations, i.e., $0.25-1.00 \mathrm{~g} \mathrm{l}^{-1}$ of plant powder, no further separations were observed. Such phenomenon, of source inoculum effect, was defined as the influence of the inoculum on the growth of its pertinent load of bacterial cells.

To confirm such phenomenon, another experiment was carried out with maize phyllosphere. This is primarily to test the pretreatment of inocula suspensions to eliminate as much as possible their contents of nutrients' traces. This pretreatment included low-speed centrifugation for the removal of coarse plant debris and high-speed centrifugation for collecting pellets of bacterial cells, together with successive washings (Supplementary methods). Results indicated highly significant differences $\left(P=2.24 \times 10^{-6}\right)$ attributed only to the inoculum pretreatment (Fig. S2). The significant decreases in counts of CFUs developed on WA not $\mathrm{AJ}$ is a clear indication on the affinity between the nutrients and the bacterial load of the inocula.
Table 1 Log CFU counts of phyllosphere bacteria of sunflower plants developed on all tested culture media over incubation time

\begin{tabular}{lll}
\hline Culture media & \multicolumn{2}{l}{ Incubation time (days) } \\
\cline { 2 - 3 } & 4 days & 9 days \\
\hline Plain water agar (WA) & $3.94 \pm 0.23^{\mathrm{d}^{*}}$ & $4.20 \pm 0.26^{\mathrm{bcd}}$ \\
Standard R2A & $4.45 \pm 0.20^{\mathrm{ab}}$ & $4.52 \pm 0.18^{\mathrm{ab}}$ \\
Autoclave-sterilized sunflower & $4.05 \pm 0.32^{\mathrm{cd}}$ & $4.39 \pm 0.29^{\mathrm{abc}}$ \\
juice (AJ) & & \\
Filter-sterilized sunflower juice (FJ) & $4.48 \pm 0.20^{\mathrm{ab}}$ & $4.60 \pm 0.15^{\mathrm{a}}$ \\
\hline
\end{tabular}

*Statistically significant differences are designated by different letters based on Tukey's Honestly Significant Differences (HSD, $P \leq 0.05$, $n=4)$

In view of the previous results obtained, we further analyzed the phyllosphere bacteria of sunflower plant (Helianthus annuus L.) using the IDC strategy to confirm the microcolonies phenomenon and specifically analyze their taxonomic affiliation. In general, counts of CFUs developed on culture media of AJ and FJ of sunflower, diluted R2A, and plain WA were in the range of log 3.9- $\log 4.6 \mathrm{~g}^{-1}$. With prolonged incubation (9 days), oneway ANOVA displayed no significant differences, and counts of CFUs developed on the plain WA were very much comparable with those developed on the conventional culture media based on plant juices (AJ and $\mathrm{FJ}$ ) or chemically synthetic nutrients (R2A) (Table 1). Again, the pervasive occurrence of microcolonies was reported on both WA and FJ. Randomly, we picked representatives of those microcolonies ( $\mu$ CFUs) for further subculturing and taxonomic identification using $16 \mathrm{~S}$ rRNA gene sequencing. Isolates derived from WA plates failed to further subculturing on plain WA. Interestingly however, all of the isolates, including those recovered from WA, successfully maintained culturability on FJ semi-solid and agar culture media for up to 4-6 subsequent generations. This is a further proof that IDC is a workable culturing strategy of potential application as a culturomic tool in future. As to the microcolonies, some retained their status ( $<1 \mathrm{~mm}$ dia.), while others developed into macrocolonies (>1 mm dia.), with varying chromogenic phenotypes. This is contrary to what have been earlier reported by previous investigations, where microcolonies were irreproducible, i.e., they were not able to grow when subcultured on the same original culture media of isolation [8, 12, 17]. It appeared that microcolonies require further and careful domestication/passage process to sustainably grow in standard petri dishes [18]. In our case, microcolonies were able not only to just survive after subculturing, but also proliferate and turn into macrocolonies when further subcultured on the related plant-only juice culture media (FJ). 


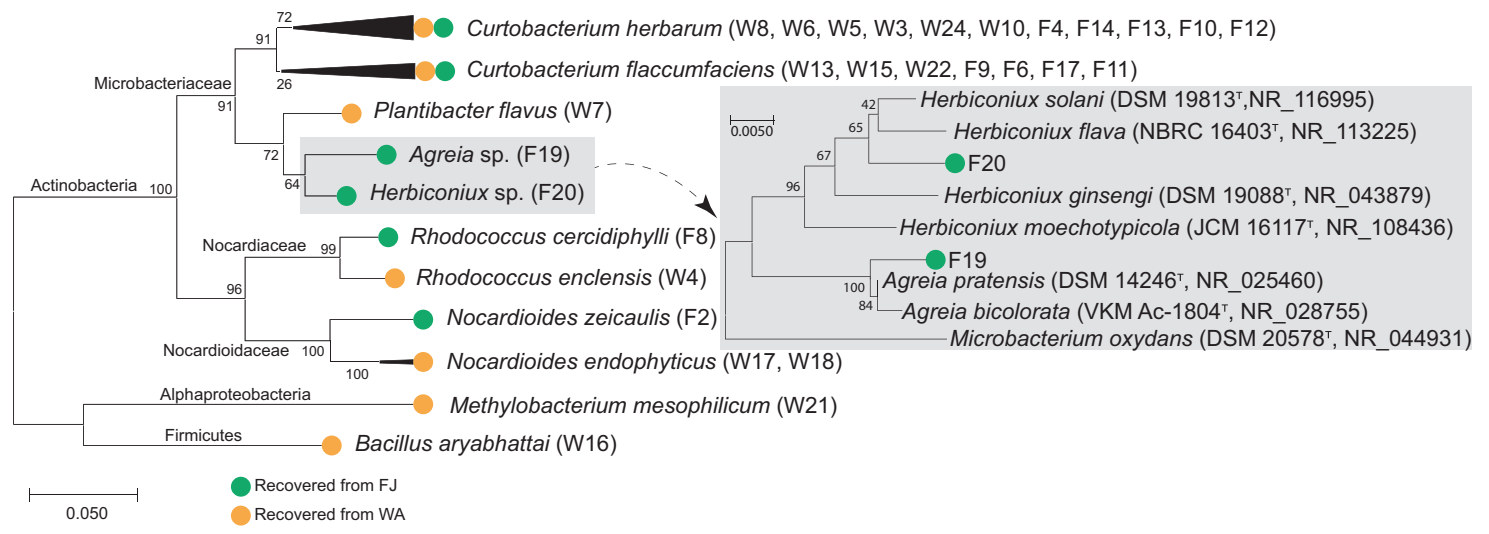

Fig. 2 Maximum likelihood (ML) phylogenetic tree of the bacterial isolates recovered from WA (15 isolates) and FJ (13 isolates) culture media, based on 16S rRNA gene sequences. The subtree includes the isolates F19 and F20 with all reported species of the genera Agreia and Herbiconiux, including Microbacterium oxydans as an outgroup. Bootstrapping was performed for each tree with 1000 replicates; the

The 16S rRNA gene sequences of secured isolates were deposited in the GenBank under the accession numbers MK100479-MK100506, and compared with the databases of GenBank (ncbi.nlm.nih.gov) and EZBioCloud (ezbiocloud.net). The analysis revealed the domination of endophytic Actinobacteria species, with single incidences of each of the phyla Proteobacteria (Methylobacterium mesophilicum) and Firmicutes (Bacillus aryabhattai). The 26 isolates of the phylum Actinobacteria represented the genera Curtobacterium spp. (18 isolates), Plantibacter spp. (1 isolate), Agreia spp. (1 isolate), and Herbiconiux spp. (1 isolate) of the family Microbacteriaceae, as well as the genus Rhodococcus spp. (2 isolates) of the family Nocardiaceae, and the genus Nocardioides spp. (2 isolates) of the family Nocardioidaceae (Fig. 2). Interestingly, the isolates F19 and F20 are most likely to represent novel species - their highest matching scores were $<98.7 \%$. This was confirmed as Maximum likelihood phylogeny, with bootstrapping of 1000 replicates, displayed significant separation of such two isolates away from all of the deposited members of the genera Herbiconiux and Agreia (Fig. 2).

Our results highlight the endophytic nature of Actinobacteria, as well as their wide occurrence in plant tissues, being widely known as a potential bio-repertoire of natural products. To facilitate their isolation and cultivation, tens of culture media were developed and enabled isolation of wide range of Actinobacteria, as described in literature (Table S1). They all contain one or more of diversified carbon and nitrogen sources, as well as growth factors, e.g., soluble starch, lignin, chitin, cellulose, glycerol, asparagine, casein, yeast extract, soil extract, humic acid, and consortia of vitamins and amino acids $[19,20]$. Culture media derived from plant materials (e.g., oatmeal medium, YIM21) are percentage of trees in which the associated taxa clustered together is shown next to the branches. Phylogenetic analyses were conducted in MEGA X. WA water agar, R2A 1/100 diluted (v/v) R2A, AJ autoclaved 1/200 diluted (v/v) sunflower juice agar, and FJ filtered 1/200 diluted (v/v) sunflower juice agar culture media

also recommended [19]. It appeared that a prerequisite of culturing fastidious Actinobacteria is to suppress fastgrowing microbes and eliminate competition of Grampositive and Gram-negative bacteria, as well as fungi. This is achieved through use of complex $\mathrm{C}$ and $\mathrm{N}$ sources, salt concentrations, $\mathrm{pH}$ levels, and appropriate antibiotics and chemicals. This is imperative to keep mining the rich store of antibiotic, active compounds, and secondary metabolites of newly isolated Actinobacteria [21, 22].

As the WA does not support growth of most prokaryotes per se [23], the reported ability of WA to recover CFU counts implies that the applied inocula contained both microbes (biomass) and nutrients (plant juice). In theory, the constitutive effect of the source inoculum on $\mathrm{CFU}$ development is considered of an intrinsic nature. When using conventional rich culture media, such effect is suppressed, i.e., being recessive. Employing the host plant materials (e.g., juices, concentrates and powders) in the preparation of plant-based culture media, as in Mourad et al. and Hegazi et al. [5, 6], the effect appears as synergic. In case of using IDC or highly diluted culture media, the effect appears as dominant. This persuades us to reconsider previous reports on the use of diluted culture media and the resulted increases in culturability $[6,24]$. We may extrapolate that such increases are most likely considered as an inoculum effect. This supports the growing interest in strategies of increasing cultivability of in situ bacterial communities by boosting the recovery of microcolonies via dilution of nutrients present in the recommended standard culture media and/or adjusting in vitro atmosphere of the surroundings $[2,17]$.

In conclusion, our results encourage the use of ultradiluted plant-only-based culture media, contained in the 
plant inoculum itself (IDC), for in vitro cultivation of the plant microbiota. The method guarantees providing realtime nutrients of the tested homologous host plants with their unique diversity, complexity, and concentrations. This strategy is a simple, practical, and convenient approach to mine for the hidden and novel members of the plant microbiota, particularly those of biotechnological potential like Actinobacteria. It is a promising culturomic tool, and highly recommended for future screening programs that require isolation of large number of isolates from diverse plants, as well as multiple analysis of other environmental microbiomes.

Acknowledgements The present work was funded by the GermanEgyptian Research Fund (GERF-STDF 5032). Hegazi acknowledges the support of Alexander von Humboldt Foundation for equipment subsidies and financing his research stays in Germany, at IGZ. Our gratitude is extended to Prof Eckhard George, the IGZ research director, for his continuous support and cooperation. The junior authors appreciate the financial aid provided by edX foundation for pursuing the online courses 7.QBWx, BIF001x BIF002x, and BIF003x, through edX platform (edx.org).

\section{Compliance with ethical standards}

Conflict of interest The authors declare that they have no conflict of interest.

Publisher's note: Springer Nature remains neutral with regard to jurisdictional claims in published maps and institutional affiliations.

Open Access This article is licensed under a Creative Commons Attribution 4.0 International License, which permits use, sharing, adaptation, distribution and reproduction in any medium or format, as long as you give appropriate credit to the original author(s) and the source, provide a link to the Creative Commons license, and indicate if changes were made. The images or other third party material in this article are included in the article's Creative Commons license, unless indicated otherwise in a credit line to the material. If material is not included in the article's Creative Commons license and your intended use is not permitted by statutory regulation or exceeds the permitted use, you will need to obtain permission directly from the copyright holder. To view a copy of this license, visit http://creativecommons. org/licenses/by/4.0/.

\section{References}

1. Libby EA, Silver PA. Harnessing undomesticated life. Nat Microbiol. 2019;4:212-3.

2. Lagier JC, Dubourg G, Million M, Cadoret F, Bilen M, Fenollar F et al. Culturing the human microbiota and culturomics. Nat Rev Microbiol 2018;16:540-50.

3. Berdy B, Spoering AL, Ling LL, Epstein SS. In situ cultivation of previously uncultivable microorganisms using the ichip. Nat Protoc. 2017;12:2232-42.

4. Sarhan MS, Patz S, Hamza MA, Youssef HH, Mourad EF, Fayez M, et al. G3 PhyloChip analysis confirms the promise of plant-based culture media for unlocking the composition and diversity of the maize root microbiome and for recovering unculturable candidate divisions/phyla. Microbes Environ. 2018;33:317-25.
5. Mourad EF, Sarhan MS, Daanaa HA, Abdou M, Morsi AT, Abdelfadeel MR, et al. Plant materials are sustainable substrates supporting new technologies of plant-only-based culture media for in vitro culturing of the plant microbiota. Microbes Environ. 2018;33:40-9.

6. Hegazi NA, Sarhan MS, Fayez M, Patz S, Murphy BR, Ruppel S. Plant-fed versus chemicals-fed rhizobacteria of Lucerne: Plantonly teabags culture media not only increase culturability of rhizobacteria but also recover a previously uncultured Lysobacter sp., Novosphingobium sp. and Pedobacter sp. PLoS ONE. 2017;12:e0180424.

7. Sarhan MS, Mourad EF, Hamza MA, Youssef HH, Scherwinski AC, El-Tahan M, et al. Plant powder teabags: a novel and practical approach to resolve culturability and diversity of rhizobacteria. Physiol Plant. 2016;157:403-13.

8. Youssef HH, Hamza MA, Fayez M, Mourad EF, Saleh MY, Sarhan MS, et al. Plant-based culture media: efficiently support culturing rhizobacteria and correctly mirror their in-situ diversity. J Adv Res. 2016;7:305-16.

9. Saleh MY, Sarhan MS, Mourad EF, Hamza MA, Abbas MT, Othman AA, et al. A novel plant-based-sea water culture media for in vitro cultivation and in situ recovery of the halophyte microbiome. J Adv Res. 2017;8:577-90.

10. Sarhan MS, Hamza MA, Youssef HH, Patz S, Becker M, ElSawey $\mathrm{H}$ et al. Culturomics of the plant prokaryotic microbiome and the dawn of plant-based culture media-A review. J Adv Res. 2019;19:15-27.

11. Gutleben J, Chaib De Mares M, van Elsas JD, Smidt H, Overmann J, Sipkema D. The multi-omics promise in context: from sequence to microbial isolate. Crit Rev Microbiol. 2018;44:212-29.

12. Overmann J, Abt B, Sikorski J. Present and future of culturing bacteria. Annu Rev Microbiol. 2017;71:711-30.

13. Nour EH, Hamza MA, Fayez M, Monib M, Ruppel S, Hegazi NA. The crude plant juices of desert plants as appropriate culture media for the cultivation of rhizospheric microorganisms. J Adv Res. 2012;3:35-43.

14. Zengler K, Toledo G, Rappe M, Elkins J, Mathur EJ, Short JM, et al. Cultivating the uncultured. Proc Natl Acad Sci USA. 2002;99:15681-6.

15. Orlewska K, Potrowska-Seget Z, Cycoń M. Use of the PCRDGGE method for the analysis of the bacterial community structure in soil treated with the cephalosporin antibiotic cefuroxime and/or inoculated with a multidrug-resistant Pseudomonas putida strain MC1. Front Microbiol. 2018;9:1387.

16. Edenborn SL, Sexstone A. DGGE fingerprinting of culturable soil bacterial communities complements culture-independent analyses. Soil Biol Biochem. 2007;39:1570-9.

17. Da Rocha UN, Andreote FD, de Azevedo JL, van Elsas JD, van Overbeek LS. Cultivation of hitherto-uncultured bacteria belonging to the Verrucomicrobia subdivision 1 from the potato (Solanum tuberosum L.) rhizosphere. J Soils Sediment. 2010;10:326-39.

18. Nichols D, Cahoon N, Trakhtenberg EM, Pham L, Mehta A, Belanger A, et al. Use of ichip for high-throughput in situ cultivation of "uncultivable" microbial species. Appl Environ Microbiol. 2010;76:2445-50.

19. Jiang Y, Li Q, Chen X, Jiang C. Isolation and cultivation methods of Actinobacteria. In: Dhanasekaran D, editor. Actinobacteria-Basics and Biotechnological Applications. 1st ed. London: IntechOpen Ltd; 2016. p. 39-57.

20. Wei W, Zhou Y, Chen F, Yan X, Lai Y, Wei C, et al. Isolation, diversity, and antimicrobial and immunomodulatory activities of endophytic actinobacteria from Tea Cultivars Zijuan and Yunkang-10 (Camellia sinensis var. assamica). Front Microbiol. 2018;9:1304. 
21. Barka EA, Vatsa P, Sanchez L, Gaveau-Vaillant N, Jacquard C, Meier-Kolthoff JP, et al. Taxonomy, physiology, and natural products of actinobacteria. Microbiol Mol Biol Rev. 2016;80:1-43.

22. Goodfellow M, Nouioui I, Sanderson R, Xie F, Bull AT. Rare taxa and dark microbial matter: novel bioactive actinobacteria abound in Atacama Desert soils. Antonie Van Leeuwenhoek. 2018;111: 1315-32.
23. Silva HSA, Romeiro RdS, Mounteer A. Development of a root colonization bioassay for rapid screening of rhizobacteria for potential biocontrol agents. J Phytopathol. 2003;151: $42-6$.

24. Armanhi JSL, de Souza RSC, Damasceno NB, de Araujo LM, Imperial J, Arruda P. A community-based culture collection for targeting novel plant growth-promoting bacteria from the sugarcane microbiome. Front Plant Sci. 2017;8:2191. 